\title{
REFLEXÕES SOBRE O PLANEJAMENTO DE UNIDADES DE TRATAMENTO INTENSIVO (UTIS) NA PERSPECTIVA DOS USUÁRIOS
} CONSIDERATIONS REGARDING THE PLANNING OF INTENSIVE CARE UNITS (ICU) UPQN
USER'S PERSPECTIVE

\author{
Patrícia Biasi Cavalcanti ${ }^{1}$, Camila Maçaneiro ${ }^{2}$, Isabella Postiglione ${ }^{3}$, Júlia Martan Nazário \\ Palma ${ }^{4}$, Julia Roberta $\mathrm{Eli}^{5}$
}

\section{RESUMO:}

O presente trabalho discute o resultado da avaliação do ambiente físico de três unidades de tratamento intensivo (UTIs) de hospitais públicos da Grande Florianópolis. Objetivou-se identificar problemas recorrentes nessas unidades, a partir da percepção ambiental de seus profissionais de saúde e também da avaliação dos pesquisadores, e refletir sobre possíveis soluções. Para tanto, foram realizadas: revisão de literatura; visitas exploratórias a duas UTIs de hospitais particulares; e aplicada a metodologia de avaliação pós-ocupação em três estudos de caso de hospitais públicos, nos quais foram empregados o mapeamento visual e a análise walkthrough. Cabe destacar que, na análise walkthrough, as três unidades não atenderam plenamente aos aspectos mínimos necessários ao funcionamento do local, os quais dizem respeito, em sua maioria, a itens previstos em resoluções e normas. Dentre os problemas recorrentes encontrados nas três UTIs avaliadas estão: subdimensionamento de ambientes; insuficiência ou inadequação de móveis e equipamentos; ambientação pouco humanizada; sobreposição indesejável de usos; problemas de conservação e de manutenção; falta de visibilidade em relação aos leitos; acabamentos inadequados; e aspectos deficitários da infraestrutura. Espera-se, através da discussão dos problemas constatados e de possíveis soluções, contribuir para a atuação profissional, para a gestão pública desses locais e para a revisão da legislação vigente.

PALAVRAS-CHAVE: Arquitetura hospitalar; Avaliação pós-ocupação; Percepção ambiental.

\section{ABSTRACT:}

The present work discusses the results of the physical environment evaluation from three Intensive Care Units (ICU) of public hospitals from the Great Florianópolis. It aimed to identify the recurring problems in these units from the environmental perception feature from their healthcare professionals and also from the researches' evaluation, and to reflect on possible solutions. Therefore, the following was conducted: literature review; exploratory visits to two private hospitals ICUs, and Post Occupancy Evaluation in three public hospitals case studies where it was applied visual mapping and walkthrough analysis. It is important to highlight that, in the walkthrough analysis, the three Units have not attended strongly the minimum necessary aspects to the local functioning, which refer, in the majority, to aspects provided in resolutions and rules. Among the reoccurring problems found in the three ICUs evaluated there are: undersizing of environments; insufficient or unqualified furniture and equipment; unfriendly ambiance; undesirable overlapping of uses; conservation and maintenance problems; lack of visibility in regard to beds; inappropriate finishing; and defective infrastructure aspects. It is expected that with the discussion of the observed problems and of possible solutions it contributes with the professional acting, public management of these places and review of current legislation.

KEYWORDS: Healthcare architecture; Post occupancy evaluation; Environmental perception.

How to cite this article:

CAVALCANTI, Patrícia Biasi et al. Reflexões sobre o planejamento de Unidades de Tratamento Intensivo UTIs - na perspectiva dos usuários. Gestão \& Tecnologia de Projetos. São Carlos, v16, n4, 2021. https://doi.org/10.11606/gtp.v16i4.178547

\section{Profa. Adjunta Departamento Expressão Gráfica UFSC \\ ${ }^{2}$ Arquiteta e urbanista graduada pela UFSC}

${ }^{3}$ Arquiteta graduada pela UNISUL. Pós-graduada em arquitetura hospitalar pelo Instituto de Ensino e Pesquisa Albert Einstein

${ }^{4}$ Arquiteta e urbanista graduada pela UFSC. Atua no escritório Studio Motirõ

${ }^{5}$ Arquiteta e urbanista graduada pela UFSC. Atua no escritório Ana Toscano Arquitetura

Fonte de Financiamento: MEC SESU e FINEP

Conflito de Interesse:

Declara não haver.

Submetido em: 25/11/2020

Aceito em:

01/05/2021 


\section{INTRODUÇÃO}

A permanência no ambiente hospitalar está, com frequência, associada a desgaste físico e emocional devido à doença e ao estresse inerente ao processo de tratamento. Além dos pacientes e acompanhantes, também os profissionais de saúde ficam continuamente sujeitos a situações de estresse, uma vez que as atividades por eles desempenhadas demandam elevado grau de atenção e de responsabilidade, além de interagirem com pacientes e acompanhantes que tendem a estar fragilizados fisicamente e psicologicamente (BAGGIO et al., 2011).

No caso de unidades de tratamento intensivo (UTIs) esse quadro se acentua, já que se trata de ambientes críticos do hospital onde se prestam cuidados a pacientes com estado de saúde muito delicado (BRASIL, 2010). Ainda que parte do desgaste emocional vivenciado nesses locais seja independente das instalações físicas, estas podem contribuir para amenizar a experiência dos usuários, quando elas oportunizam atributos ambientais como o contato com a natureza (KAPLAN; KAPLAN, 1989) e outras formas de distrações positivas, além de suporte social e controle do ambiente (ULRICH et al., 1991; ULRICH et al., 2010). Assim, o espaço físico do hospital tanto pode contribuir para mitigar situações de estresse e restauração da capacidade de atenção, quanto pode contribuir para tornar a experiência hospitalar ainda mais negativa, caso seja ele mesmo fonte de estresse ambiental.

Pretende-se neste trabalho contribuir para o tema identificando problemas frequentes em três UTIs avaliadas, a partir de estudo centrado na compreensão da percepção ambiental dos seus profissionais de saúde e na avaliação técnica dos pesquisadores. Busca-se também refletir sobre possíveis soluções para esses problemas, sejam elas: no âmbito da revisão da regulamentação vigente que orienta o planejamento de estabelecimentos assistenciais de saúde - RDC 50 (BRASIL, 2002) -, no âmbito da atuação profissional de arquitetos e no âmbito da gestão pública. Espera-se contribuir para o desenvolvimento de projetos futuros de UTIs que sejam cada vez mais responsivas a necessidades, atividades, expectativas e anseios de pacientes, acompanhantes e profissionais de saúde. Para tanto, o instrumento (checklist) elaborado para a análise walkthrough de três estudos de caso pode dar suporte à avaliação de outras unidades, em diferentes localidades do país, e com isso contribuir para a atuação de gestores da área da saúde.

\section{FUNDAMENTAÇÃO TEÓRICA}

A UTI proporciona suporte para pacientes em estado crítico de saúde (NASCIMENTO; TRENTINI, 2004), buscando contribuir para a recuperação de suas funções vitais (BONILHA et al., 2015). 0 ambiente costuma dispor de avançada tecnologia biomédica e fornece atendimento especializado à saúde vinte e quatro horas por dia (DA SILVA; FERREIRA, 2008). Segundo a RDC 50 (BRASIL, 2002), dentre as atividades realizadas na UTI incluem-se: executar e registrar assistência médica e de enfermagem intensivas ao paciente, prestar apoio diagnóstico, monitorar e prestar assistência respiratória, prestar assistência nutricional e fisioterapêutica, além de prover informações aos acompanhantes e familiares.

A configuração da unidade pode variar de acordo com seu perfil: UTI adultos, UTI neonatal, UTI pediátrica, UTI cardiológica, UTI neurológica, UTI traumas, entre outras. Porém, alguns critérios projetuais são comuns aos diferentes perfis como seu posicionamento em relação a outros setores do hospital, ambientes que compõem seu programa de necessidades e estratégias visando à sua humanização.

No que se refere ao posicionamento da UTI, é prioritária sua proximidade ou seu acesso fácil à Emergência e ao Centro Cirúrgico, a partir dos quais os pacientes costumam ser 
encaminhados, e precisa ainda estar próxima ou ter acesso fácil à Farmácia do hospital ou dispor de uma Farmácia satélite, para prover a medicação necessária ao local (SAMPAIO; CEZAR, 2004; RUNGTA et al., 2020). Também é recomendável o acesso fácil à Unidade de Patologia Clínica, visando dar suporte à realização de exames laboratoriais, e ao Banco de Sangue (DALMASSO, 2005; RUNGTA et al., 2020). Rungta et al. (2020) destacam ainda a importância de que a unidade tenha apenas uma entrada e saída, com monitoramento contínuo, garantindo a segurança de todos.

No que se refere ao programa de necessidades de uma UTI, os ambientes que a integram são: recepção e admissão, sala de espera, sala administrativa, área clínica, posto de enfermagem e área de serviços de enfermagem, apoio logístico (rouparia, almoxarifado, sala de utilidades, depósito de material de limpeza e depósito de equipamentos e materiais) e conforto pessoal (quarto de plantão, banheiro do quarto de plantão, sanitários com vestiários para funcionários, sanitários para pacientes, copa e área de estar) (SAMPAIO; CEZAR, 2004; CARVALHO, 2014). A resolução RDC 50 (BRASIL, 2002) prevê como ambientes não obrigatórios a área para prescrição médica e a sala de entrevistas, esta última destinada a conversas privadas entre familiares e a equipe médica, além de prever obrigatoriamente a sala de higienização e preparo de material, a qual pode ser dispensada apenas se a atividade ocorrer na Central de Material Esterilizado (CME).

O principal local da UTI é a área clínica, onde ficam os leitos dos pacientes, usualmente organizado em ambientes coletivos de tratamento e/ou em quartos privativos. Ainda que ambas as configurações apresentem diferentes vantagens e desvantagens, estudos indicam que quartos privativos tendem a: reduzir a disseminação de infecções hospitalares, possibilitar a realização de uma maior variedade de atividades de atendimento e gerar um impacto positivo no paciente (CHAUDHURY; MAHMOOD; VALENTE, 2005). Em estudo de Dettenkofer et al. (2004) o planejamento de UTIs com quartos privativos e a maior disponibilidade de área por leito foram dois dentre três aspectos ambientais que mais impactaram na redução de infecções hospitalares. Segundo Rashid (2006) e Thompson et al. (2012) quartos privativos proporcionam para os pacientes melhores condições de privacidade visual e acústica, maior controle sobre o ambiente (ajustes na iluminação, som, disposição do mobiliário,...), melhor qualidade do sono, e melhor comunicação entre estes e os profissionais de saúde, aumentando o nível de satisfação do paciente. Rashid (2006) também destaca que quartos privativos bem planejados permitem acomodar acompanhantes, que são uma imensurável fonte de informação sobre a saúde de pacientes que chegam ao local inconscientes, além de que eles proporcionam suporte social durante o processo de recuperação.

O estudo de Caruso et al. (2014) em uma UTI oncológica apontou maior prevalência de delírio em pacientes em leitos de áreas coletivas de tratamento do que aqueles admitidos em leitos privativos, decorrente de fatores como: maior exposição a ruído, perturbação do sono, estresse, falta de privacidade, falta de controle sobre a iluminação, pior interação pacientefamiliar e maior movimentação de pessoas no local.

O estudo de Shepley, Harris e White (2008) aponta para níveis mais elevados de satisfação e níveis mais reduzidos de estresse também para a equipe de saúde na configuração de UTIs organizadas em quartos privativos. Entre as principais justificativas estão: o menor impacto da morte de um paciente sobre a equipe de saúde, por oportunizarem a escolha de como participar desse momento de sofrimento dos familiares; e a ocorrência de menos conflitos entre os profissionais de saúde, por reduzir conversas informais em áreas comuns.

Ainda no que se refere à opção por quartos privativos, Rashid (2006) destaca a tendência para planejá-los com área suficiente para permitir: o acesso livre a todas as laterais do leito hospitalar, a acomodação dos diversos equipamentos médicos e a realização das atividades 
de atendimento em condições de conforto, além de favorecer a acomodação dos acompanhantes. 0 autor incentiva a utilização de colunas de energia e estativas para acomodar os equipamentos médicos e ao mesmo tempo liberar o acesso à cabeceira do leito, sem encostá-la em qualquer parede, além do uso de portas de vidro para facilitar a visualização do paciente.

Mesmo em UTIs organizadas majoritariamente em ambientes coletivos de tratamento, recomenda-se prever ao menos 1 quarto de isolamento para cada 10 leitos (BRASIL, 2002), para a acomodação de pacientes com doenças infectocontagiosas e pacientes imunodeprimidos (CARVALHO, 2014). Wagner et al. (2019) recomendam ainda a previsão de um quarto desocupado dentro da UTI com sistema de pressurização e filtro de ar semelhante ao de salas cirúrgicas, para oportunizar a realização de procedimentos invasivos urgentes, em condições adequadas de assepsia.

Assim, os benefícios proporcionados tanto aos pacientes quanto aos profissionais de saúde têm estimulado o planejamento de UTIs com quartos privativos, mais frequente em países desenvolvidos, mas também no Brasil, principalmente na rede privada de saúde, a exemplo do Hospital Israelita Albert Einstein, BP Mirante e Hospital Sírio Libanês. situados em São Paulo, capital.

Assim como a área clínica, o posto de enfermagem é outro ambiente fundamental das UTIs, o qual deve assegurar visibilidade direta e acesso fácil para todos os leitos (SAMPAIO; CEZAR, 2004; LU; ZIMRING, 2012; LU et al., 2014; TAVARES; SANTOS; BURNSZTYN, 2014). A visibilidade entre pacientes e profissionais de saúde melhora a comunicação entre eles e impacta na redução dos seguintes aspectos como: na taxa de mortalidade, no risco de quedas dos pacientes, no tempo de espera por atendimento, nas distâncias percorridas, aumentando o tempo dedicado à assistência ao paciente (LU; ZIMRING, 2012; LU et al., 2014). Lu et al. (2014) apontam que mesmo diferenças sutis na visibilidade dos leitos a partir do posto de enfermagem podem ter efeitos importantes nos resultados clínicos e na mortalidade de pacientes, confirmando a sua relevância para o planejamento de UTIs. Por esse motivo, Carvalho (2014) sugere que o posto de enfermagem seja central e aberto, com leitos dispostos ao seu redor em formato de "U", e com divisórias fixas ou retráteis que proporcionem autonomia e privacidade ao paciente, mesmo em áreas coletivas de tratamento. Hamilton (2001 apud DALMASSO, 2005) considera ideais as configurações semicircular e circular, pela visibilidade que proporcionam aos leitos. No entanto, as UTIs com áreas coletivas em formato retangular são mais difundidas.

Em unidades com quartos privativos, para melhorar as condições de visibilidade aos pacientes e de audição dos aparelhos médicos é possível dispor de centrais de monitoramento eletrônico nos postos de enfermagem (RASHID, 2006; SHEPLEY; HARRIS; WHITE, 2008). Outra estratégia é a descentralização do posto de enfermagem em subestações menores, aproximando os profissionais dos pacientes por eles monitorados e reduzindo deslocamentos (SHEPLEY; HARRIS; WHITE, 2008). Em estudo de Seo, Choi e Zimring (2011) que comparou diferentes leiautes de UTI, a unidade avaliada com posto de enfermagem central proporcionou melhor visibilidade geral, isto é, um profissional conseguia enxergar um número maior de leitos. Porém, na unidade projetada exclusivamente com quartos privativos e postos de enfermagem descentralizados - um pequeno posto a cada 2 quartos - constataram-se melhores condições de visibilidade específica, isto é, entre o profissional e os leitos dos pacientes por ele atendidos. Assim, para os autores, a excelente visibilidade dos postos de enfermagem descentralizados associada ao posicionamento estratégico das medicações, permitem reduzir o deslocamento do corpo de enfermagem e maximizar o tempo dedicado ao atendimento ao paciente, mesmo considerando-se que a unidade com quartos privativos avaliada possuía maior área total. 
Sampaio e Cezar (2004) recomendam que cada posto de enfermagem sirva ao número máximo de 12 leitos, com espaço suficiente para acomodar confortavelmente a equipe de saúde e dar suporte ao exercício de suas funções. Para o preparo dos medicamentos é necessário dispor de uma área de serviços de enfermagem junto ao posto, equipada com balcões, pia e lavatório, refrigerador e iluminação adequada, entre outros (SAMPAIO; CEZAR, 2004; BRASIL, 2013).

No que se refere à higiene pessoal, a RDC 50 (BRASIL, 2002) recomenda pelo menos um sanitário para pacientes em geral em UTIs, e caso haja mais de um, que $30 \%$ deles sejam acessíveis e que atendam a NBR 9050 (ABNT, 2020). Segundo Carvalho (2014), todos os sanitários (sem chuveiro) ou banheiros (com chuveiro) de pacientes dessa unidade deveriam assegurar acesso à cadeira de rodas, tendo em vista que uma parcela significativa desses usuários tem condições reduzidas de mobilidade ou dependem do auxílio de terceiros para sua utilização. Rashid (2006) aponta as seguintes razões para que todos os quartos privativos tenham seu próprio banheiro: reduz o risco de contaminação pelo compartilhamento do local, reduz a necessidade de transportar dejetos pelos profissionais de saúde, e proporciona condições mais confortáveis para pacientes que têm boas condições de mobilidade. 0 autor destaca ainda a importância da proximidade do banheiro ao leito. Especificamente para o quarto de UTI destinado à realização de procedimentos invasivos de urgência, Wagner et al. (2019) entendem que a disponibilidade de banheiro ou sanitário não é recomendável, pois se tornaria uma fonte de contaminação.

Outros aspectos do planejamento da unidade contribuem positivamente para a experiência dos usuários, e consequentemente para a humanização do local. Dentre eles, está a presença de janelas com vista para o exterior, proporcionando distrações positivas e orientação em relação ao tempo e ciclo circadiano, recursos importantes para pacientes acamados por longos períodos (HAMILTON, 2001 apud DALMASSO, 2005; RASHID, 2006; THOMPSON et al., 2012). Tavares, Santos e Burnsztyn (2014) recomendam que, quando possível, as UTIs disponham de espaços voltados especificamente para áreas verdes. Ferrer (2012) recomenda que o leito seja posicionado de modo a favorecer a visualização pela equipe de saúde e ao mesmo tempo assegurar a visão tanto para o céu quanto para os jardins, através de janelas com peitoris adequados ao campo de visão do paciente acamado. Segundo Shepley et al. (2012) a iluminação natural e o visual para o exterior também se mostraram recursos importantes para os profissionais de saúde, pois causaram a redução no nível de absenteísmo da equipe de uma UTI que foi reformada comparativamente aos dados obtidos em sua configuração espacial anterior.

Além da iluminação natural e dos visuais para o exterior, a iluminação artificial e os níveis sonoros costumam ser críticos em UTIs. Os elevados níveis luminosos proporcionados pelas luminárias e equipamentos médicos, assim como o elevado nível de ruído de alarmes, respiradores, aparelhos de sucção, entre outros, impactam negativamente no bem-estar dos usuários. Segundo Donchin e Seagull (2002) o alto nível de ruídos típico de UTIs dificulta a atuação do corpo de saúde, que tem uma capacidade limitada para captar e interpretar os diversos estímulos ambientais, e que necessita dar respostas rápidas durante o processo de atendimento ao paciente. Walder et al. (2000) apontam ainda que o excesso de sons e luzes é a causa mais frequente de distúrbios do sono em pacientes de UTI, o que prolonga a estadia no local, impacta na taxa de mortalidade e eleva os custos da internação hospitalar. Dentre as principais recomendações visando à melhoria do sono do paciente estão: proporcionar um nível constante e baixo o suficiente de iluminação que possibilite o atendimento à saúde e a visualização de todos os aparelhos médicos sem impedir o repouso (WALDER et al., 2000; FERRER, 2012); transferir todos os aparelhos médicos que forem possíveis para fora do quarto do paciente, pressupondo-se que a UTI esteja organizada em quartos privativos; e, instalar moduladores sonoros nos aparelhos para emitirem menos ruído no período noturno 
(WALDER et al., 2000). Estudo experimental de Luetz et al. (2016) em uma UTI reformada constatou a redução dos níveis de ruído e seu impacto sobre o sono do paciente decorrentes principalmente de duas estratégias projetuais: a criação de uma "sala de trabalho" (working room) para alocar equipamentos médicos fora do quarto e para permitir a observação do paciente por visores de vidro, sem necessidade de acessar o ambiente; e o fechamento automático das portas de entrada dos quartos. Engwall et al. (2015) propõem que os pacientes possam regular os níveis luminosos, para criar condições adequadas para o repouso e o bem-estar, além de permitir variações na iluminação ao longo do dia e da noite.

Visando ainda contribuir para o bem-estar dos pacientes de UTI, são recomendações presentes na literatura dispor de: relógio, calendário e condições para personalização do local (THOMPSON et al., 2012); e espaços adequados para a acomodação dos familiares, tais como salas de espera, salas de entrevistas - opcionais segundo a RDC 50 (BRASIL, 2002) -, dormitório para pernoite, e sala para meditação (THOMPSON et al., 2012; RUNGTA et al., 2020). Thompson et al. (2012) apontam também para os benefícios psicológicos das visitas de animais de estimação a pacientes internados na UTI, desde que os ambientes sejam higienizados antes e depois da sua realização, e, quando possível, haja um ambiente específico para elas.

Também é relevante que a UTI proporcione ambientes de apoio às necessidades dos profissionais de saúde, contribuindo para a redução de quadros de estresse, como quarto de plantão e copa. 0 quarto de plantão, destinado ao repouso, deve ser silencioso, com acesso restrito, e deve dispor, no mínimo, de cama e mobiliário de apoio (balcão, armários, etc.), embora outras amenidades sejam desejáveis como televisão ou local para estudos. A copa deve dispor de equipamentos de cozinha (forno de micro-ondas, pia e refrigerador) e mobiliário (armário, bancada, mesa, cadeiras...) sendo, muitas vezes, um dos principais locais de descanso e ao mesmo tempo de socialização da equipe de saúde. Souza e Ferreira (2010) destacam a importância de prever um ambiente de estar de uso exclusivo da equipe de saúde, opcional segundo a RDC 50 (BRASIL, 2002). Rungta et al. (2020) recomendam ainda a previsão de uma pequena biblioteca para a equipe médica, além de computadores e acesso à internet.

Assim, o planejamento da UTI deve objetivar sua funcionalidade tanto quanto o conforto dos pacientes, dos acompanhantes e da equipe de saúde. Para tanto, ele deve ser centrado nas necessidades dos usuários e proporcionar boas condições de atuação e de interação entre eles. Um ambiente com tais características pode contribuir para um processo de recuperação da saúde mais rápido e para uma experiência mais positiva no ambiente hospitalar por todos.

\section{METODOLOGIA}

O estudo, de natureza qualitativa e exploratória, baseia-se na aplicação de instrumentos da metodologia de avaliação pós-ocupação, e em pesquisas das áreas de psicologia ambiental e arquitetura hospitalar. Por isso, reflete a opinião dos usuários das unidades estudadas, além da avaliação técnica dos pesquisadores. 0 projeto de pesquisa deste trabalho foi aprovado na Plataforma Brasil com o número CAAE: 24452317.0.0000.0121.

Dado o contexto, e em especial o estado de saúde dos pacientes, não foi possível aplicar os métodos de coleta de dados diretamente com esse público. A coleta de dados foi feita com os profissionais de saúde - médicos, enfermeiros, técnicos de enfermagem, fisioterapeutas, fonoaudiólogos -, por terem necessidades críticas em relação ao local, por terem conhecimento aprofundado de toda a unidade, bem como por poderem expressar as necessidades espaciais dos pacientes. Na definição da amostra optou-se por focar nos profissionais que utilizam cotidianamente a unidade, e assim foram excluídos os 
profissionais da construção civil e os gestores externos, visto que as chefias médica e de enfermagem das unidades participaram do estudo. Também se optou por não consultar os acompanhantes dos pacientes, uma vez que as visitas normalmente ocorrem em horários restritos, nem sempre coincidindo com os horários limitados de permanência dos pesquisadores no local.

0 trabalho se fundamenta em: revisão da literatura, visitas exploratórias a UTIs de duas instituições da rede privada, e na avaliação de três estudos de caso em hospitais públicos, nos quais foram aplicados os instrumentos de análise walkthrough e mapeamento visual.

Para a revisão de literatura, recorreu-se a periódicos científicos, dissertações, teses, livros, além de documentos disponíveis on-line.

Em seguida foram realizadas visitas exploratórias a unidades de tratamento intensivo da Grande Florianópolis, de duas instituições da rede privada de saúde, sendo uma delas inaugurada em 2014 e a outra reformada em 2015. As visitas exploratórias tinham duração de até duas horas, e foram conduzidas por um profissional de saúde de cada unidade, o qual era entrevistado, com base em um roteiro semiestruturado. Também foram realizadas observações diretas e assistemáticas dos ambientes de cada unidade e realizados registros fotográficos pelos pesquisadores.

Os três estudos de caso ocorreram em UTIs da rede pública de saúde, sendo: a primeira delas reformada em 2005, contando atualmente com 18 leitos; a segunda reformada em 2014, contando com 10 leitos; e a terceira reformada em 2018, contando com 10 leitos também. A primeira técnica aplicada foi a análise walkthrough, na qual é realizada a observação de todos os ambientes associada à entrevista a usuários enquanto se percorre o local (RHEINGANTZ et al., 2009). Para tanto, foi elaborado um checklist específico para avaliação de UTIs, que abrangeu os aspectos mínimos a serem considerados em seu planejamento, projeto ou obra, sendo parte dos itens previstos na resolução RDC 50 (BRASIL, 2002) e na norma NBR 9050 (ABNT, 2020), além daqueles aspectos considerados fundamentais presentes na citada revisão de literatura ou observados nas visitas exploratórias. 0 checklist refletiu tanto o ponto de vista dos pesquisadores quanto dos profissionais de saúde. Criada no Excel (Tabela 1), a planilha de checklist apresentou aproximadamente 350 itens de avaliação para os quais foi possível atribuir nota, e sua aplicação em cada unidade era de aproximadamente 2 a 3 horas. As perguntas sobre aspectos técnicos e diretamente observáveis do local foram respondidas pelos pesquisadores, como, por exemplo, sobre o atendimento às áreas mínimas de cada ambiente previstas na resolução. Aos profissionais de saúde foram direcionadas as perguntas sobre a adequação de ambientes, leiaute e mobiliário às atividades neles realizadas. Desse modo, foi otimizada a participação dos profissionais de saúde na avaliação, sem, contudo, deixar de incorporar sua percepção ambiental. As perguntas respondidas pelos usuários receberam peso maior, evidenciando a importância desses aspectos da avaliação e do seu olhar sobre o ambiente. 0 modo de elaboração desta planilha se assemelha ao já utilizado em outra pesquisa previamente realizada em Unidades de Urgência e Emergência hospitalares (CAVALCANTI et al., 2019).

Participaram da análise walkthrough os seguintes profissionais: um médico e a enfermeira chefe da primeira unidade avaliada; a enfermeira chefe da segunda; e a enfermeira chefe além de outra enfermeira da terceira. Outros profissionais de saúde também participaram da análise walkthrough respondendo a algumas perguntas em relação aos ambientes específicos que estavam utilizando no momento em que se percorria o local. Porém, foram participações parciais no que se refere ao instrumento, apenas visando complementar algumas das respostas anteriores. 
Tabela 1. Parte do checklist da análise walkthrough referente a apenas um ambiente.

Fonte: acervo dos autores.

\begin{tabular}{|c|c|c|c|c|c|c|}
\hline 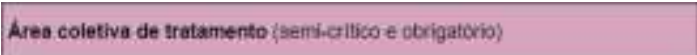 & Lei: & Peso & Nota & $\begin{array}{l}\text { Nota } \\
\text { final }\end{array}$ & Comentikrios & $\begin{array}{l}\text { Tpo de } \\
\text { resposta }\end{array}$ \\
\hline $\begin{array}{l}\text { Apresenta a area minima segundo a RDC } 50\left(2,0 \mathrm{~m}^{2} \text { por leito com dlstancia }\right. \\
\text { de } 1 \mathrm{~m} \text { entre, paredes e ielto, excelo cabeceira, de } 2 \mathrm{~m} \text { entre leitos e } 1,2 \mathrm{~m} \text { a } \\
\text { partir do pe da lello)? }\end{array}$ & FOC 50 & 3 & & & & $\sin$ \\
\hline $\begin{array}{l}\text { O ambiente esta prowimo de barheiro, para lso do pacientes, que seja } \\
\text { acessival de acorto com a noma (NBR 9050)? }\end{array}$ & NBR $\$ 050$ & 2 & & & & $\sin$ \\
\hline 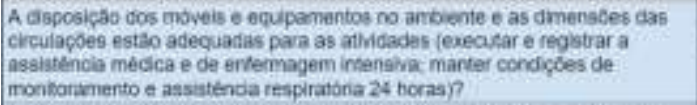 & $\begin{array}{l}\text { SOMASUS } \\
\text { (FOC 50) }\end{array}$ & 3 & & & & siniparcia \\
\hline $\begin{array}{l}\text { Dapoe do mobilario e equpamerio mirimo para as abvidades reailzadas } \\
\text { (cama hosptasar, mena para reteicalo, cadeira, mesa de cabeceira)? }\end{array}$ & $\begin{array}{l}\text { SOMASUS } \\
\text { (ROC 50) }\end{array}$ & 3 & & & & onjperciar \\
\hline A ltuminaçdo esta acequaca para a realizapso das abvidades? & & 2 & & & & $\sin$ \\
\hline Pcasul revestimentos adequados? & $\begin{array}{l}\text { SOMASUS } \\
\text { (ROC SO) }\end{array}$ & & & & & \\
\hline 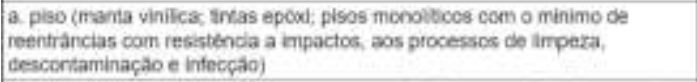 & & 0,33 & & & & $\sin$ \\
\hline $\begin{array}{l}\text { b. rodape (materiais que fociliem o processo de impeza e nat criem cantos } \\
\text { de acumbio de realdios) }\end{array}$ & & 0,33 & & & & $\sin$ \\
\hline $\begin{array}{l}\text { c. parede (tintas epoxi, pvt, ou todos tipos de pintura destinadas a areas } \\
\text { molhadas; laminado metaminico) }\end{array}$ & & 0,33 & & & & sin \\
\hline 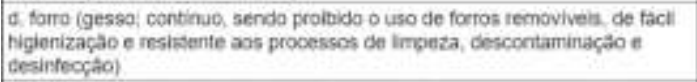 & & 0,33 & & & & $\sin$ \\
\hline e. moveis (laninedo melaminico; mdr, mateciais lisos e resisternos a limpeza) & & 0,33 & & & & $\sin$ \\
\hline $\begin{array}{l}\text { f. portas (laminado melaminico; tirtas a tase de cleo, esmaite sintefico, } \\
\text { maneriais ligce e resistentes o limpeza) }\end{array}$ & & 0,33 & & & & $\sin$ \\
\hline Pcssul regua de gases para todos ca leflos de paciente? & & 3 & & & & $\sin$ \\
\hline O anbiente encontra-se em bom estado de manutengta? & & 2 & & & & sinporcia \\
\hline Total & & 20.0 & & & $0,00 \%$ & \\
\hline
\end{tabular}

A opção por atribuir notas e pesos ao espaço físico segue o modelo já desenvolvido pelo National Health System para o ASPECT (NHS, 2009b) e o AEDET (NHS, 2009a), instrumentos elaborados para que qualquer pessoa possa avaliar a configuração ambiental de unidades de saúde na Inglaterra. No entanto, os instrumentos de avaliação ingleses focam na qualidade dos ambientes de saúde visando assegurar sua excelência, enquanto a planilha desenvolvida para essa avaliação, com perguntas totalmente distintas e organizadas por ambientes, prioriza aspectos técnicos, funcionais e de humanização considerados mínimos e indispensáveis ao bom funcionamento do local.

Os dados obtidos por meio da análise walkthrough resultaram em percentuais que permitiram compreender qual proporção dos aspectos considerados mínimos ou essenciais foram atendidos por cada unidade como um todo e por cada um dos seus ambientes. Assim, foi possível identificar os ambientes e as unidades mais problemáticas e realizar uma análise qualitativa.

Nos três estudos de caso também foi aplicado o mapeamento visual com os seguintes profissionais de saúde: médicos, enfermeiros, técnicos de enfermagem, fisioterapeutas, psicólogos, entre outros. Para tanto, a eles foram entregues formulários (Figura 1) com plantas baixas da unidade e foi solicitado que identificassem aspectos positivos e negativos do local, associando-os à respectiva planta. Assim, a técnica permitiu evidenciar quais os aspectos mais representativos da percepção ambiental dos usuários e associá-los aos locais específicos da unidade, sem direcionamento prévio para nenhum resultado ou resposta. Considerando-se os vários turnos de profissionais de saúde de cada UTI e buscando maximizar o número de participantes, os formulários eram deixados no local com a chefia da unidade, e junto a eles era colocada uma folha introdutória explicando seu preenchimento, além do termo de consentimento livre e esclarecido. Os formulários ficaram nas unidades entre 1 e 3 semanas e eram recolhidos quando a chefia acreditava que já havia alcançado o número máximo possível de respondentes. Participaram da aplicação do mapeamento visual: 21 profissionais de saúde da primeira unidade avaliada, 12 da segunda e 18 da terceira. 


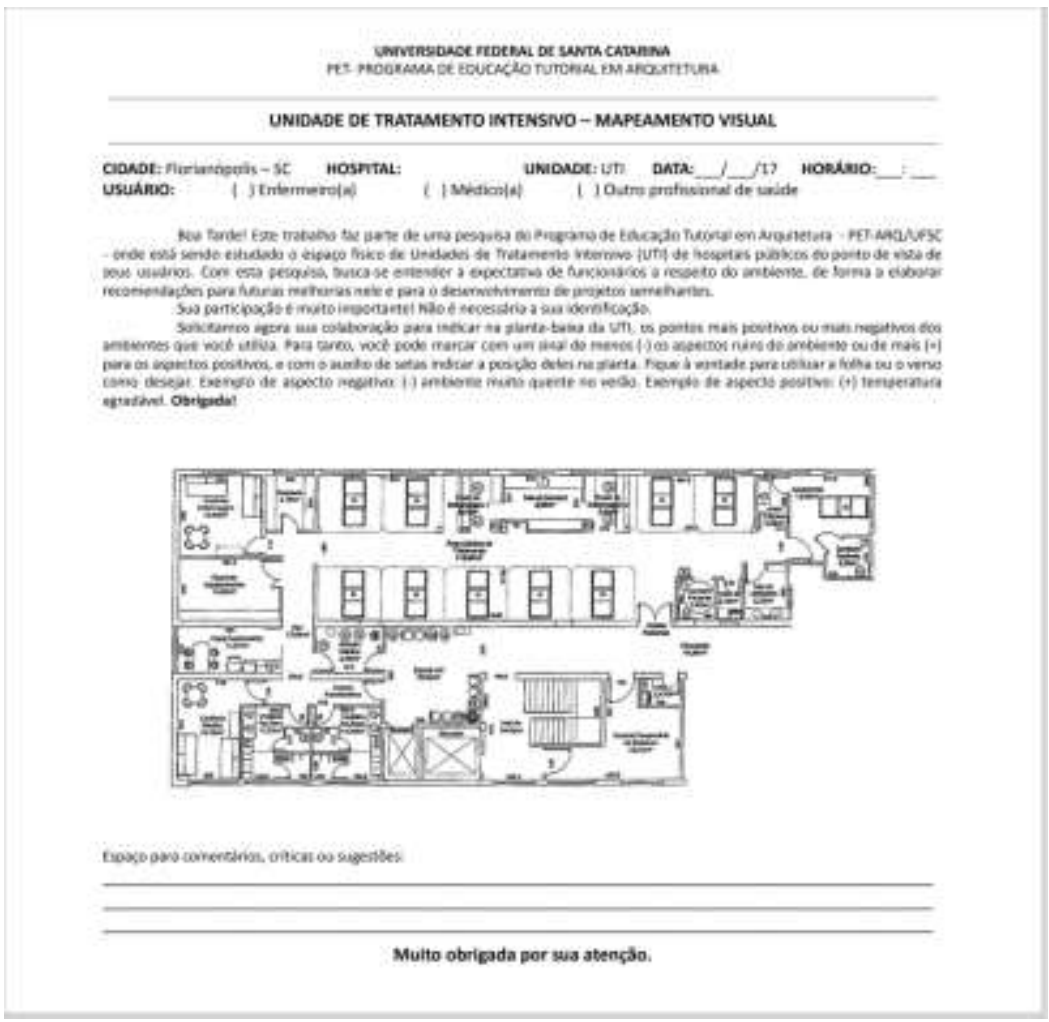

Figura 1. Formulário de mapeamento visual.

Fonte: acervo dos autores.

Os dados obtidos através do mapeamento visual foram categorizados por meio de análise de conteúdo, identificando-se os aspectos ambientais positivos e negativos de cada uma das unidades, recorrentes na percepção dos respondentes, além dos ambientes mais bem avaliados e também os mais deficitários. Assim, também no que se refere ao mapeamento visual foi adotada uma abordagem qualitativa para o tratamento dos dados.

Por fim, foi elaborada uma matriz de descobertas de cada uma das unidades avaliadas, na qual foram sintetizados os principais resultados obtidos em cada uma delas por meio de tópicos e de imagens, associados à planta baixa do local.

\section{RESULTADOS E DISCUSSÃO}

A revisão de literatura permitiu compreender: o que são UTIs, qual seu perfil de usuários, quais as atividades que nelas se realizam, configurações ambientais recorrentes, e aspectos ambientais relativos à sua humanização. Assim, a revisão de literatura proporcionou uma visão abrangente sobre o tema e embasou o posterior trabalho de campo.

As visitas exploratórias às UTIs privadas possibilitaram a compreensão inicial sobre sua configuração espacial e funcionamento. Dentre os aspectos positivos que se destacaram, e que se distinguem das UTIs públicas avaliadas, estão: o seu excelente estado de conservação e manutenção; a utilização de monitores eletrônicos complementando as condições de visualização dos pacientes a partir dos postos de enfermagem; e o fato de que uma das unidades se organizava exclusivamente com quartos privativos, e outra delas com boxes com divisórias fixas entre eles, executados em alvenaria e vidro, proporcionando melhores condições de privacidade e assepsia para pacientes do que a utilização de cortinas hospitalares. Dentre os aspectos negativos observados, comuns também às UTIs públicas avaliadas, estão: quarto de plantão da equipe médica com qualidade ambiental sensivelmente superior ao quarto da equipe de enfermagem; copas pequenas e com poucos atrativos; e poucas possibilidades de distração positiva para pacientes em uma das unidades. 
A análise walkthrough permitiu constatar que as três UTIs públicas avaliadas nos estudos de caso obtiveram resultados totais similares, que variaram de aproximadamente $68 \%$ a $70 \%$. Assim, a análise walkthrough revelou que nem todos os aspectos mínimos necessários ao seu funcionamento foram plenamente atendidos nas três unidades. De qualquer modo, tais percentuais sugerem que as UTIs públicas avaliadas não se encontravam em uma situação crítica no que se refere à configuração espacial.

0 mapeamento visual, por sua vez, permitiu aos usuários avaliar livremente aspectos positivos e negativos de cada ambiente, e o resultado de sua aplicação revelou que duas das unidades apresentaram uma prevalência de avaliações negativas. Assim, embora o resultado obtido pelas unidades na análise walkthrough não fosse tão baixo, os resultados do mapeamento visual confirmaram haver diversos aspectos do ambiente considerados insatisfatórios na percepção da equipe de saúde, e que poderiam ser aprimorados no ambiente físico de cada uma delas para torná-las mais responsivas aos usuários.

Dentre os aspectos positivos mencionados pelos usuários na análise walkthrough e no mapeamento visual destacaram-se: o posicionamento estratégico das três unidades perto do Centro Cirúrgico dos hospitais, embora longe das unidades que realizam exames em duas das UTIs; e o fato de duas das três unidades terem sido reformadas há menos de 7 anos, contribuindo para uma imagem positiva do ambiente.

Os problemas recorrentes das UTIs públicas avaliadas em ordem decrescente de frequência foram: subdimensionamento de ambientes; insuficiência ou inadequação de móveis e equipamentos; escassez de elementos que contribuíssem para a humanização do local; problemas de conservação e manutenção de acabamentos e móveis; sobreposição de usos; visibilidade insuficiente da equipe de saúde para todos os leitos; acabamentos inadequados; e aspectos deficitários da infraestrutura.

O subdimensionamento de ambientes foi o problema mais recorrente. Na maioria das situações, os ambientes atenderam à área mínima solicitada pela RDC 50 (BRASIL, 2002), mas ainda assim foram considerados pequenos para a realização de todas as atividades necessárias. Esse problema ocorreu principalmente em: postos de enfermagem, áreas de prescrição médica, área de serviços de enfermagem, áreas coletivas de tratamento - boxes com leitos dos pacientes e áreas de circulação -, e quartos de isolamento. No posto de enfermagem e áreas afins (área de prescrição médica e área de serviços), todas as unidades atenderam à área mínima normatizada, embora não fosse considerado suficiente em duas das três UTIs avaliadas, as quais tinham entre 9 e 15 leitos ativos dentre os leitos disponíveis. Como consequência não havia, no posto de enfermagem e adjacências, área suficiente para acomodar todos os profissionais de saúde (Figura 2), além de gerar conflitos de fluxos nas circulações, o que se destacou negativamente na percepção dos usuários. Uma alternativa para o problema, a ser avaliada em estudos futuros, é que o cálculo da área mínima do posto de enfermagem e ambientes afins previsto na resolução RDC 50 (BRASIL, 2002), que atualmente é fixo e similar para diferentes setores hospitalares, fosse proporcional ao tamanho da unidade, à quantidade de leitos de UTI ou à quantidade de profissionais de saúde a serem acomodados. Alguns boxes das áreas coletivas de tratamento das três UTIs e os quartos de isolamento de duas das UTIs também foram considerados subdimensionados, ainda que atendessem à área mínima normatizada. Além disso, a dificuldade de manobrar as macas nas circulações e junto aos leitos dos pacientes foi identificada nas três UTIs. A pesquisa revelou a importância de se realizar estudos específicos sobre o leiaute da área clínica de UTIs, considerando a acomodação de todos os equipamentos e as dimensões dos modelos mais novos de leitos hospitalares, de forma a confirmar se a área mínima prevista na resolução realmente é suficiente para acomodá-los adequadamente e favorecer a manobra de macas. Independentemente de tais estudos, recomenda-se aos arquitetos estarem atentos ao 
dimensionamento das áreas livres ao redor dos leitos e áreas de circulação, de forma a não apenas atender à área mínima normatizada, mas a efetivamente favorecer a circulação de macas. Em algumas unidades, a sala de utilidades e a rouparia não atenderam à área mínima prevista na RDC 50 (BRASIL, 2002). A área da rouparia, no entanto, não foi considerada um problema na percepção da equipe de saúde, uma vez que ela pode ser substituída por armário roupeiro. A sala de utilidades, por outro lado, não atendeu à área mínima necessária para acomodar o armazenamento temporário de resíduos em duas das UTIs, embora o ambiente também fosse utilizado para esse fim. Nesse caso, o descumprimento da área mínima prevista na RDC 50 (BRASIL, 2002) resultou na presença de cestos de roupa suja (hampers) e carrinhos de lixo em ambientes inadequados para essa função (Figura 3). Por fim, um sanitário destinado ao público de uma das unidades e banheiros destinados aos pacientes de outras duas unidades não atenderam plenamente aos critérios de acessibilidade relacionados ao seu dimensionamento. Segundo a RDC 50 (BRASIL, 2002), apenas 30\% dos banheiros de unidades de internação, incluindo UTIs, necessitam ser adaptados para o uso por cadeirantes, o que faz com que essas unidades não estejam descumprindo a legislação, embora a pesquisa de campo tenha revelado ser insuficiente. Como também aponta Carvalho (2014), estudos futuros poderiam confirmar a indicação de ter um percentual maior de banheiros e sanitários de pacientes adaptados ao cadeirante em UTIs.
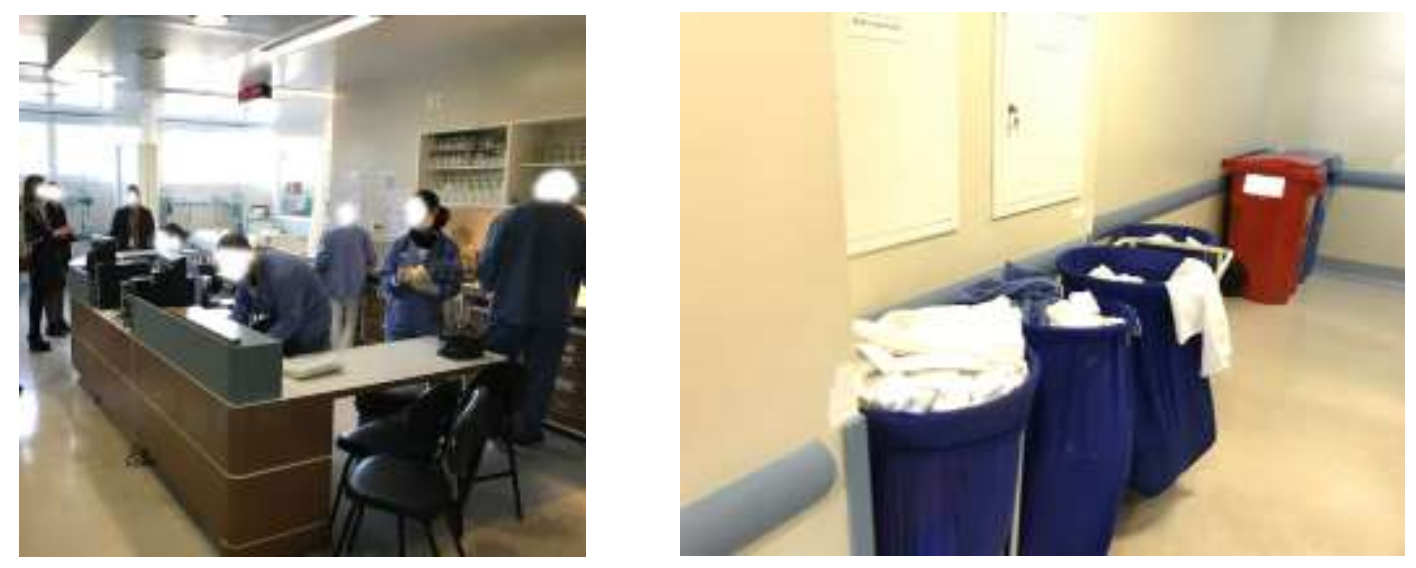

O segundo aspecto mais recorrente foi relativo a mobiliário e equipamentos. Observou-se a falta de: armários para a guarda de pertences pessoais dos pacientes; cadeiras para acomodar todos os visitantes na sala de espera em horários de maior fluxo; prateleiras e armários para organização de produtos e objetos guardados no DML e na sala de utilidades; móveis mais confortáveis para acomodar os familiares nos ambientes utilizados como salas de entrevistas; guarda-volumes suficientes em vestiários da equipe de saúde; móveis e equipamentos de suporte ao conforto nos quartos de plantão da equipe de enfermagem e de técnicos de enfermagem; e ausência de geladeira em uma das copas. Nenhum desses problemas acima mencionados comprometia a realização do atendimento à saúde na unidade, porém a disponibilidade desses móveis e equipamentos revelou-se desejável para o conforto de pacientes, visitantes e funcionários. Alguns desses resultados vão ao encontro da revisão de literatura no que se refere à importância de dar suporte à acomodação de acompanhantes (THOMPSON et al., 2012; RUNGTA et al., 2020) e ao conforto da equipe de saúde (SOUZA; FERREIRA, 2010; RUNGTA et al., 2020).

0 terceiro aspecto mais recorrente foi relativo à humanização do espaço físico das UTIs avaliadas. Dentre os problemas constatados incluem-se em ordem decrescente de frequência: a falta de privacidade acústica e visual proporcionada pelas cortinas hospitalares que dividem os boxes dos leitos de pacientes; a ausência de visão para o exterior, devido à falta de
Figura 2. Posto de enfermagem com elevado número de profissionais para acomodar.

Fonte: acervo dos autores.

Figura 3. Cestos de roupa suja e carrinhos de lixo nos corredores.

Fonte: Acervo dos Autores. 
janelas ou à presença de janelas com peitoris muito elevados para os pacientes acamados; a ausência de áreas de estar para a equipe de saúde; a escassez de distrações positivas (televisão, artes, livros,...); e a ambientação institucional das três unidades. Percebe-se, em hospitais norte-americanos, na rede privada de saúde do Brasil, bem como nos estudos sobre o tema (CHAUDHURY; MAHMOOD; VALENTE, 2005; DETTENKOFER et al., 2004; RASHID, 2006; THOMPSON et al. , 2012; CARUSO et al., 2014; SHEPLEY; HARRIS; WHITE, 2008), a tendência para a priorização de quartos privativos nas UTIs. Alterar a resolução vigente no país tornando obrigatório o uso de quartos privativos certamente a enrijeceria. Porém, sempre que possível, recomenda-se que ao planejar novas unidades ou reformar existentes, os arquitetos considerem a adoção de quartos privativos ou ao menos de boxes com divisórias rígidas. E, almejando dispor de visão para o exterior, é importante que os arquitetos posicionem a área clínica da UTI junto ao perímetro externo da edificação, o que não ocorria em uma das unidades avaliadas.

As três UTIs avaliadas não apresentaram área de estar para a equipe de saúde, ambiente recomendado por Souza e Ferreira (2010), porém opcional segundo a RDC 50 (BRASIL, 2002). Em todas as unidades a copa e os quartos para os plantonistas funcionavam como espaços de acolhimento da equipe de saúde, ainda que, devido à sua configuração ambiental, dimensões e mobiliário, não fossem adequados para realizar outras atividades, em seus momentos de intervalo, como socializar, ler, assistir à televisão, utilizar um computador, entre outros. Em especial, os quartos de plantão de uso exclusivo da equipe de enfermagem e de técnicos de enfermagem apresentaram uma ambientação ainda mais austera do que os quartos de plantão da equipe médica, oportunizando essencialmente o repouso.

Os demais elementos relativos à humanização, como distrações positivas e integração interior-exterior, destacados também na revisão de literatura (HAMILTON, 2001 apud DALMASSO, 2005; RASHID, 2006; THOMPSON et al., 2012; TAVARES; SANTOS; BURNSZTYN, 2014), poderiam ser facilmente proporcionados, especialmente quando considerados em momentos iniciais do processo projetual.

0 quarto aspecto mais recorrente diz respeito à sobreposição de usos em alguns ambientes das UTIs avaliadas, gerando conflitos na utilização do espaço. Nesse quesito destacaram-se os problemas relativos à ausência de sala de uso exclusivo para entrevistas, visto que nas três unidades avaliadas essa atividade ocorria na sala de espera ou na sala administrativa. Nas duas unidades em que as entrevistas ocorriam na sala de espera, os participantes do estudo apontaram falta de privacidade para as conversas entre a equipe de saúde e os familiares dos pacientes. Por esse motivo, em uma das unidades avaliadas optou-se por fechar com chave uma das salas de espera para que ela funcionasse exclusivamente como sala de entrevistas, desprovendo a unidade desse ambiente. Na UTI cuja sala administrativa também acomodava a função de sala de entrevistas, os participantes alegaram que o mobiliário não era adequado para criar condições acolhedoras de diálogo com os familiares. Ainda que a sala de entrevistas não seja obrigatória segundo a resolução RDC 50 (BRASIL, 2002), na percepção dos participantes do estudo, ela é muito importante, coincidindo com a recomendação de Thompson et al. (2012), sendo desejável aos arquitetos incorporá-la ao programa de necessidades sempre que possível.

Em duas unidades avaliadas, um dos boxes de atendimento ao paciente situado na área coletiva de tratamento foi desativado para funcionar como depósito temporário de grandes equipamentos como poltronas, suportes de soro, entre outros (Figura 4). Em uma dessas UTIs não havia depósito de equipamentos e materiais, e na outra UTI o depósito foi considerado subdimensionado pelos usuários. A inadequação e insuficiência da área para armazenamento de equipamentos reduziu a capacidade de atendimento dessas duas UTIs, e também comprometeu a ambiência do local, visto que os equipamentos ficavam acumulados 
e expostos na área coletiva de tratamento. Foram observadas ainda, com uma ocorrência apenas, as seguintes sobreposições de uso: quarto de plantão dos técnicos de enfermagem funcionando também como vestiário da equipe de saúde, o que gerou bastante insatisfação nos técnicos, visto que a movimentação intensa de profissionais no local comprometia seu repouso; depósito de equipamentos e materiais funcionando também como área de serviços, porém sem infraestrutura adequada para esse fim (bancada, lavatório para as mãos, pia,...); uma rouparia de grande porte funcionando também como copa, acomodando bebedouro e refrigerador; e o vestiário dos funcionários acumulando também a função de banheiro do quarto de plantão, subdimensionado para atender a toda a demanda.

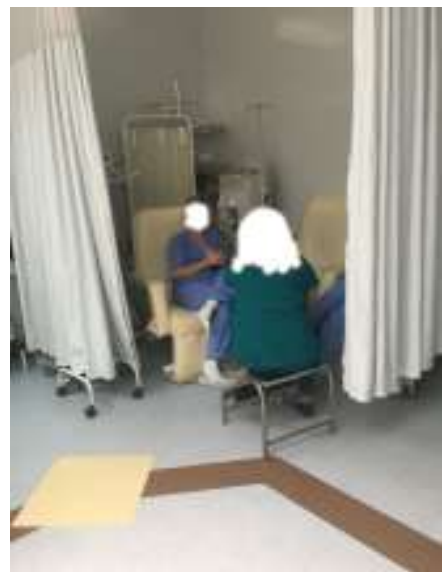

Nas três unidades avaliadas, desprovidas da sala de higienização e preparo de equipamentos, a atividade em questão, que também poderia ocorrer na CME (BRASIL, 2002), era feita no próprio leito do box de atendimento, assim que ele era desocupado, ou no depósito de equipamentos e materiais. Em uma das UTIs, uma nova higienização era feita assim que um novo paciente fosse ocupar o local. Em nenhuma das unidades a ausência do ambiente foi percebida pela equipe de saúde como sendo problemática ou como gerando sobreposição de usos.

0 quinto aspecto mais recorrente refere-se à conservação e manutenção de acabamentos de piso, parede, forro e mobiliário. Embora as três unidades tenham sido reformadas ao longo do tempo, e, de modo geral, as condições de acabamento fossem boas, em alguns ambientes específicos de duas delas observaram-se problemas de: mofo, infiltrações, e buracos em divisórias de gesso acartonado e no forro; e móveis velhos e/ou danificados. Embora o gesso acartonado seja indicado para a execução de paredes de ambientes de saúde em geral, ele se mostrou danificado pelo impacto das macas em duas da UTIs, razão pela qual é recomendável sua manutenção periódica pelos gestores, assim como também de outros acabamentos, móveis e equipamentos.

O sexto aspecto mais recorrente foi a pouca visibilidade de alguns leitos específicos a partir do posto de enfermagem. Em geral, referiam-se a: leitos de quartos de isolamento, ou leitos posicionados nos cantos da área coletiva de tratamento ou ainda a leitos cuja visualização era dificultada por pilares mal posicionados. Nas três UTIs avaliadas o posto de enfermagem estava posicionado centralmente em relação aos leitos, de forma a favorecer o contato visual entre a equipe de saúde e o paciente, embora não tenha sido condição suficiente. Considerando-se a importância de que a equipe de saúde tenha ótimas condições de contato visual para todos os pacientes, uma solução complementar ao posicionamento estratégico do posto de enfermagem e dos leitos, prevista na literatura (RASHID, 2006; SHEPLEY; HARRIS; WHITE, 2008) e verificada nas UTIs particulares visitadas, é o monitoramento eletrônico (com central de monitores) complementando a observação direta. Outra opção, adotada mais comumente em projetos de UTI norte-americanas, e prevista na literatura (SHEPLEY;
Figura 4. Leito desativado funcionando como depósito de equipamentos e estar da equipe de saúde.

Fonte: acervo dos autores. 
HARRIS; WHITE, 2008; SEO; CHOI; ZIMRING, 2011), é o planejamento de subestações de enfermagem, distribuídas ao longo da área coletiva de tratamento ou junto a cada grupo pequeno de quartos privativos. Assim, os profissionais de saúde são posicionados em locais mais próximos dos pacientes que estão observando.

O sétimo aspecto recorrente que se destacou na análise se refere à infraestrutura das UTIs estudadas. Duas delas não apresentavam leitos suficientes para acomodar pacientes destinados à hemodiálise, limitação que se destacou na percepção da equipe de saúde no mapeamento visual. Outro problema constatado, relativo à infraestrutura, foi a falta de chuveiro em uma das UTIs, pois só dispunha de um único sanitário para os pacientes da área coletiva de tratamento. Na percepção da equipe de saúde, esse aspecto dificultava a realização da higiene pessoal dos pacientes, forçando todos a receberem banho no leito, independentemente de sua condição de mobilidade e saúde, acarretando sobrecarga para os profissionais e limitando a privacidade e a realização da atividade pelo paciente. Segundo a RDC 50 (BRASIL, 2002) é possível dispor apenas de sanitário na UTI, porém, na percepção dos profissionais de saúde consultados, seria desejável dispor de ao menos um banheiro para os pacientes dentro da UTI, equipado com chuveiro. Estudos futuros podem confirmar essa demanda, pois também foi possível verificar que as diferentes UTIs atendiam a diferentes perfis de paciente. Nesta UTI que dispunha apenas de sanitário, sem chuveiro, o perfil dos pacientes era de maior mobilidade, sendo que parte deles teria condições de utilizar o banheiro com certa autonomia. Em outra UTI avaliada, boa parte dos pacientes decorria de trauma (acidentes graves), com raros casos em que eles teriam condições de utilizar um chuveiro com autonomia. Assim, estudos futuros podem confirmar a demanda de banheiros dependendo do perfil da UTI. A presente pesquisa aponta a relevância de se prever ao menos um banheiro com chuveiro para pacientes da UTI, criando-se condições mais cômodas para profissionais de saúde e mais privativas para pacientes.

Por fim, um dos quartos de isolamento de uma das UTIs não apresentava banheiro ou sanitário. Mesmo não sendo obrigatório em quartos de isolamento de UTI, na percepção da equipe de saúde, a falta de banheiro ou sanitário se apresentou como um problema porque pacientes com doenças infectocontagiosas que utilizassem esse quarto de isolamento teriam que circular pela unidade para acessá-lo, expondo todos os demais usuários ao risco de contaminação. Nesse caso a revisão da resolução vigente - RDC 50 (BRASIL, 2002) se faz desejável, coincidindo com a recomendação de Rashid (2006).

Também no que se refere à infraestrutura, nessa mesma UTI, as dimensões de um dos elevadores não permitiram acomodar adequadamente as macas hospitalares de modelos mais recentes, comprometendo, na percepção da equipe de saúde, o transporte dos pacientes em macas nesse bloco do hospital.

0 penúltimo item recorrente se refere à presença de acabamentos não mais recomendados para estabelecimentos assistenciais de saúde (BRASIL, 2002), como o uso de granito em bancadas de sanitários e de banheiros, e a falta de pisos antiderrapantes nesses mesmos ambientes.

O último problema se refere à comunicação visual, ponto comum em duas das três unidades avaliadas. A ausência de sinalização suficiente para orientar os visitantes sobre como chegar à UTI e de sinalização suficiente dentro da unidade identificando os seus ambientes foi apontada pelos profissionais de saúde, mas não se caracterizou como um problema tão expressivo quanto os demais mencionados. 


\section{CONSIDERAÇÕES FINAIS}

Parte dos estudos sobre a configuração ambiental de UTIs, especialmente aqueles realizados fora do país, foca em aspectos específicos como a análise comparativa de diferentes leiautes para a unidade, a visibilidade dos leitos, ou o conforto acústico e lumínico. 0 presente trabalho, por sua vez, apresenta uma análise exploratória e abrangente da configuração ambiental de diferentes UTIs e seus principais problemas, a partir da percepção de profissionais de saúde e da avaliação dos pesquisadores. Dentre os principais resultados desta pesquisa, distintos da revisão de literatura, destacaram-se o subdimensionamento de diversos ambientes, a sobreposição indesejável de usos e os problemas de manutenção e conservação. Parte das distinções deve-se ao fato de que um dos instrumentos utilizados na avaliação baseava-se nos aspectos mínimos necessários ao funcionamento do local. No que se refere ao subdimensionamento, cabe observar que as edificações avaliadas têm décadas de existência, ao longo das quais a demanda de atendimento aumentou consideravelmente sem que houvesse um aumento proporcional no número de leitos de UTI na cidade, pressionando a estrutura de saúde existente. Assim, o trabalho contribui para o tema ao refletir sobre a problemática no contexto local. Já diversas recomendações presentes na literatura, de grande relevância para o planejamento de unidades futuras, não puderam ser aqui avaliadas, como sua organização exclusivamente em quartos privativos, visto que nos três estudos de caso a área clínica era um ambiente coletivo de tratamento ao qual se somavam alguns quartos de isolamento. Cabe ainda observar que os resultados do presente estudo complementam e corroboram a fundamentação teórica.

Além de identificar problemas frequentes em UTIs dos hospitais públicos estudados, na percepção de seus usuários, foi possível refletir sobre soluções em diferentes âmbitos: na atuação de arquitetos, orientando ações projetuais a serem tomadas; na atuação de gestores; e na revisão da legislação vigente - RDC 50 (BRASIL, 2002).

No que se refere ao âmbito da atuação de arquitetos, seguem as recomendações: dimensionar postos de enfermagem e área clínica em relação ao número de usuários e ao leiaute de mobiliário e equipamentos, não apenas restringindo-se ao atendimento da metragem mínima prevista na resolução; posicionar os postos de enfermagem de forma a assegurar a plena visibilidade para todos os leitos, ou a utilizar sistema de monitoramento por câmeras ou, ainda, considerar a utilização de postos de enfermagem menores, descentralizados e posicionados próximos aos leitos; priorizar a adoção de boxes privativos com divisórias fixas em áreas coletivas de tratamento ou de quartos privativos; planejar a sala de utilidades dimensionando-a para acomodar a função de depósito temporário de lixo, como usualmente ela de fato acomoda; prever, sempre que possível, um ambiente exclusivo para sala de entrevistas; planejar adequadamente os depósitos de equipamentos e materiais, evitando o acúmulo desses objetos em locais inapropriados; assegurar a humanização das unidades por meio de soluções como integração interior-exterior, distrações positivas e ambientação amigável e acolhedora; dialogar com a equipe de saúde e gestores durante o planejamento para verificar a quantidade necessária de leitos de hemodiálise; priorizar banheiro (com chuveiro) ao invés de sanitário para atender à área coletiva de tratamento.

Alguns dos problemas constatados referem-se à atuação de gestores e órgãos públicos e se relacionam a: necessidade de assegurar a manutenção preditiva, preventiva e corretiva regular e periódica de acabamentos e mobiliário em geral, contribuindo para a assepsia e para a imagem do ambiente; e incluir no processo de gestão o projeto de interiores de todos os ambientes, para que seja possível dispor de mobiliário que otimize o aproveitamento de espaços exíguos e que assegure a ambiência acolhedora. 
Ao longo do trabalho, a solução de alguns problemas identificados está relacionada à revisão da RDC 50 (BRASIL, 2002). No entanto, como os resultados se baseiam na avaliação de três estudos de caso de UTIs da rede pública de saúde de uma localidade específica, pesquisas futuras com uma amostra maior de unidades avaliadas, que reflitam a realidade de diferentes localidades do país, são recomendadas para confirmar ou refutar as considerações de revisão da RDC 50 (BRASIL, 2002). São elas: alterar a área mínima necessária para postos de enfermagem, área de serviços e área de prescrição médica, tornando-os proporcionais ao número de leitos da UTI ou à quantidade de profissionais de saúde a serem acomodados; rever a área mínima por leito na área coletiva de tratamento das UTIs e nos quartos de isolamento, a partir de estudos aprofundados sobre o leiaute e os fluxos nesses locais; ter todos os sanitários ou banheiros de uso de pacientes adequados a critérios de acessibilidade ou assegurar uma parcela maior que 30\%; tornar obrigatória a previsão de um banheiro para cada quarto de isolamento de UTIs, de forma a reduzir o risco de transmissão de doenças infectocontagiosas.

Dentre as contribuições do trabalho destacamos ainda a planilha de análise walkthrough elaborada, a qual se pretende disponibilizar on-line futuramente junto ao arquivo do relatório final da pesquisa na plataforma ISSU. A planilha pode ser aplicada por profissionais da construção civil, profissionais de saúde e gestores para avaliar UTIs existentes, permitindo identificar possíveis limitações do espaço físico no que se refere à legislação vigente ou aos aspectos mínimos necessários ao seu funcionamento. A planilha também pode ser aplicada na avaliação de projetos em desenvolvimento, sendo um checklist para conferência de todos os aspectos necessários ou desejáveis. Por fim, a planilha pode ser utilizada por órgãos da administração pública para avaliar sistematicamente e comparar os resultados de unidades em diferentes localidades do país, visto que seu sistema de pontuação permite identificar aquelas com problemas mais significativos e que demandam projetos de reforma ou ampliação com maior urgência.

\section{Agradecimentos}

Agradecemos à Secretaria de Educação Superior (Sesu) do Ministério de Educação (MEC) e ao Fundo Nacional de Desenvolvimento da Educação (FNDE) pelo financiamento dado ao Grupo PET Arquitetura e Urbanismo da UFSC, visto que na época da realização desta pesquisa, a professora e três das arquitetas autoras eram membros deste Programa.

\section{Referências bibliográficas}

ASSOCIAÇÃO BRASILEIRA DE NORMAS TÉCNICAS - ABNT. NBR 9050/2020: Acessibilidade a edificações, mobiliário, espaços e equipamentos urbanos. Rio de Janeiro: ABNT, 2020.

BAGGIO, Maria Aparecida et al. Privacidade em unidades de terapia intensiva: direitos do paciente e implicações para a enfermagem. Revista Brasileira de Enfermagem, v. 64, n. 1, p. 25-30, 2011. Disponível em: https://www.scielo.br/scielo.php?pid=S003471672011000100004\&script=sci_arttext . . DOI:< http://dx.doi.org/10.1590/S003471672011000100004>. Acesso em: 1 nov. 2005.

BONILHA, Larissa Gomes et al. Sentimentos e emoções vivenciados em unidade de terapia intensiva: a influência no cuidado clínico do enfermeiro. Revista de Enfermagem UFPE (online), Recife, n. 9, p. 8636-8642, jun. 2015. DOI: <10.5205/reuol.7061-61015-5-SM0906supl201502>. Acesso em: 1 nov. 2005. 
BRASIL. Ministério da Saúde. Agência Nacional de Vigilância Sanitária. RDC no 50 de 21 de fevereiro de 2002. Regulamento técnico para planejamento, programação, elaboração e avaliação de projetos físicos de estabelecimentos assistenciais de saúde. Diário Oficial República Federativa do Brasil, Poder Executivo, Brasília, DF, 21 fev. 2002.

BRASIL. Ministério da Saúde. Agencia Nacional de Vigilância Sanitária. RDC no 7 de 24 de fevereiro de 2010. Dispõe sobre os requisitos mínimos para funcionamento de Unidades de Terapia Intensiva e dá outras providências. Diário Oficial República Federativa do Brasil, Poder Executivo, Brasília, DF, 24 fev. 2010.

BRASIL. Ministério da Saúde. Secretaria-Executiva. Departamento de Economia e Desenvolvimento. Internação e apoio ao diagnóstico e terapia (reabilitação). Brasília : Ministério da Saúde, 2013. Brasil.

CARUSO, Pedro et al. ICU architectural design affects the delirium prevalence: a comparison between single-bed and multibed rooms. Critical Care Medicine, v. 42, n. 10, p. 2204-2210, 2014. Disponível em: https://journals.Iww.com/ccmjournal/Abstract/2014/10000/ICU_Architectural_Design_Affects_the _Delirium.7.aspx. DOl:< http://dx.doi.org/10.1097/ccm.0000000000000502>. Acesso em: 19 jan. 2021.

CARVALHO, Antônio Pedro Alves de. Introdução à arquitetura hospitalar. Salvador: UFBA, FA, GEAhosp, 2014.

CAVALCANTI, Patrícia Biasi et al. Avaliação pós-ocupação de unidades de emergência hospitalares de Florianópolis: problemas recorrentes e possíveis soluções. Ambiente Construído (online), Porto Alegre, v. 19, n.2, p. 171-186, 2019. DOI: <https://doi.org/10.1590/s167886212019000200315>. Acesso em: 6 ago. 2019.

CHAUDHURY, Habib; MAHMOOD, Atiya; VALENTE, Maria. Advantages and Disadvantages of SingleVersus Multiple-Occupancy Rooms in Acute Care Environments: A Review and Analysis of the Literature. Environment and Behavior, [s.I.], v. 37, n. 6, p.760-786, 2005. DOI: <http://dx.doi.org/10.1177/0013916504272658>. Acesso em: 1 nov. 2005.

DA SILVA, Rafael Celestino; FERREIRA, Márcia de Assunção. Um deslocamento do olhar sobre o conhecimento especializado em enfermagem: debate epistemológico. Revista Latino-Americana de Enfermagem, $\quad$ v. 16, n. 6, 2008. Disponível em: https://www.redalyc.org/pdf/2814/281421896017.pdf. Acesso em: 1 nov. 2005.

DALMASSO, Gabriela Liuzzi. A relação entre espaço e saúde: uma contribuição da Arquitetura para a humanização da Unidade de Tratamento Intensivo. 2005. 162 f. Dissertação (Mestrado) Programa de Pós-graduação em Arquitetura, Universidade Federal do Rio de Janeiro, Rio de Janeiro, 2005.

DETTENKOFER, Markus et al. Does the architecture of hospital facilities influence nosocomial infection rates? A systematic review. Infection Control \& Hospital Epidemiology, v. 25, n. 1, p. $21-$ 25, 2004. Disponível em: https://www.cambridge.org/core/journals/infection-control-and-hospitalepidemiology/article/abs/does-the-architecture-of-hospital-facilities-influence-nosocomialinfection-rates-a-systematic-review/52A1605C66DC3C23B23BFEBDB42F1E6B.

DOI:<doi:10.1086/502286>. Acesso em: 19 jan. 2021.

DONCHIN, Yoel; SEAGULL, F. Jacob. The hostile environment of the intensive care unit. Current Opinion in Critical Care, v. 8, n. 4, p. 316-320, 2002. Disponível em: https://journals.Iww.com/cocriticalcare/Abstract/2002/08000/The_hostile_environment_of_the_intensive_care_unit.8.aspx.

Acesso em: 17 jan. 2021. 
ENGWALL, Marie et al. Lighting, sleep and circadian rhythm: An intervention study in the intensive care unit. Intensive and Critical Care Nursing, v. 31, n. 6, p. 325-335, 2015. Disponível em: https://www.sciencedirect.com/science/article/pii/S0964339715000439.DOI:

<https://doi.org/10.1016/j.iccn.2015.07.001>. Acesso em: 17 jan. 2021.

FERRER, Mário. Manual da Arquitetura das Internações Hospitalares. 1a ed. Rio de Janeiro: Rio Books, 2012.

KAPLAN, Rachel; KAPLAN, Stephen. The experience of nature: A psychological perspective. Cambridge: Cambridge University Press, 1989.

LU, Yi; ZIMRING, Craig. Can intensive care staff see their patients? An improved visibility analysis methodology. Environment and Behavior, v. 44, n. 6, p. 861-876, 2012. DOI: < https://doi.org/10.1177/0013916511405314>. Acesso em: 20 jan. 2021.

LU, Yi et al. Patient visibility and ICU mortality: a conceptual replication. HERD: Health Environments Research \& Design Journal, v. 7, n. 2, p. 92-103, 2014. DOI:< https://doi.org/10.1177/193758671400700206>. Acesso em: 4 abr. 2021.

LUETZ, Alawi et al. Feasibility of noise reduction by a modification in ICU environment. Physiological Measurement, v. 37, n. 7, p. 1041-1055, mai. 2016. Disponível em: https://iopscience.iop.org/article/10.1088/0967-3334/37/7/1041/meta. DOI: <http://doi.org/10.1088/0967-3334/37/7/1041>. Acesso em: 06 abr. 2021.

NASCIMENTO, Eliane Regina Pereira do; TRENTINI, Mercedes. O cuidado de enfermagem na unidade de terapia intensiva (UTI): teoria humanística de Paterson e Zderad. Revista LatinoAmericana de Enfermagem. v.12, n. 2, p. 250-257, 2004. Disponível em: https://www.scielo.br/pdf/rlae/v12n2/v12n2a15.pdf. DOI:< https://doi.org/10.1590/S010411692004000200015>. Acesso em: 1 nov. 2005.

NATIONAL HEALTH SYSTEM. AEDET Evolution: Achieving Excellence Design Evaluation Toolkit Instructions, Scoring and Guidance. 2005a. Disponível em: http://www.dh.gov.uk/en/Procurementandproposals/Publicprivatepartnership/Privatefinanceinitia tive/ InvestmentGuidanceRouteMap/DH_4132945. Acesso em: 31 mar. 2009a.

NATIONAL HEALTH SYSTEM. ASPECT: Staff and Patient Environment Calibration Toolkit 21. 2005b. Disponível em: http://www.dh.gov.uk/en/Publicationsandstatistics/Publications/ PublicationsPolicyAndGuidance/ DH_082087. Acesso em: 31 mar. 2009b.

RASHID, Mahbub. A decade of adult intensive care unit design: a study of the physical design features of the best-practice examples. Critical Care Nursing Quarterly, v. 29, n. 4, p. 282-311, $2006 . \quad$ Disponivel em: https://journals.Iww.com/ccnq/Abstract/2006/10000/A_Decade_of_Adult_Intensive_Care_Unit_D esign_A.3.aspx. Acesso em: 18 jan. 2021

RHEINGANTZ, Paulo A. et al. Observando a Qualidade do Lugar: procedimentos para a avaliação pós-ocupação. Rio de Janeiro: Ed. Viana \& Mosley, 2009.

RUNGTA, Narendra et al. Indian society of critical care medicine experts committee consensus statement on ICU planning and designing, 2020. Indian Journal of Critical Care Medicine, v. 24, n. Suppl 1, p. 43-60, 2020. Disponível em: https://www.ncbi.nlm.nih.gov/pmc/articles/PMC7085818/. Acesso em: 16 jan. 2021. 
SAMPAIO, Edilene Vitorino; CEZAR, Maria de Fátima. Unidade de Tratamento Intensivo. In: CARVALHO, Antônio Pedro Alves de. Arquitetura de Unidades Hospitalares. Salvador: Virgínia Oliveira, 2004. p. 107-115.

SEO, Hyun-Bo; CHOI, Young-Seon; ZIMRING, Craig. Impact of hospital unit design for patientcentered care on nurses' behavior. Environment and Behavior, v. 43, n. 4, p. 443-468, 2011. DOI:< https://doi.org/10.1177/0013916510362635>. Acesso em: 19 jan. 2021.

SHEPLEY, Mardelle McCuskey; HARRIS, Debra D.; WHITE, Robert. Open-bay and single-family room neonatal intensive care units: caregiver satisfaction and stress. Environment and Behavior, v. 40, n. 2, p. 249-268, 2008.DOI: < https://doi.org/10.1177/0013916507311551>. Acesso em 19 jan. 2021.

SHEPLEY, Mardelle McCuskey et al. The impact of daylight and views on ICU patients and staff. HERD: Health Environments Research \& Design Journal, v. 5, n. 2, p. 46-60, 2012. DOI:< https://doi.org/10.1177/193758671200500205 >. Acesso em: 3 abr. 2021.

SOUZA, Kátia Maria Oliveira de; FERREIRA, Suely Deslandes. Assistência humanizada em UTI neonatal: os sentidos e as limitações identificadas pelos profissionais de saúde. Ciência e saúde coletiva, v. 15, p. 471-480, 2010. DOl:<https://doi.org/10.1590/S1413-81232010000200024>. Disponível em: https://www.scielo.br/pdf/csc/v15n2/v15n2a24.pdf. Acesso em: 10 nov. 2012.

TAVARES, Daniela Prado; SANTOS, Mauro César de Oliveira; BURNSZTYN, Ivani. Considerações sobre o projeto de espaços para o cuidado crítico. In: VI CBDEH - Congresso Brasileiro para o Desenvolvimento do Edifício Hospitalar, 2014, Florianópolis. Anais do VI CBDEH - Congresso Brasileiro para o Desenvolvimento do Edifício Hospitalar. Florianópolis: ABDEH, 2014. v. 1, p. 45-50. Disponível em: https://document.onl/documents/anais-vi-cbdeh.html. Acesso em: 20 jan. 2021.

THOMPSON, Dan R. et al. Guidelines for intensive care unit design. Critical Care Medicine, v. 40, n. 5, p. 1586-1600, 2012 Disponível em: https://journals.Iww.com/ccmjournal/fulltext/2012/05000/Guidelines_for_intensive_care_unit_de sign_.26.aspx. DOI: <10.1097/CCM.0b013e3182413bb2>. Acesso em: 16 jan. 2021.

ULRICH, Roger S. et al. Stress recovery during exposure to natural and urban environments. Journal of environmental Psychology, v. 11, n. 3, p. 201-230, 1991.

ULRICH, Roger S. et al. A conceptual framework for the domain of evidence-based design. HERD: Health Environments Research \& Design Journal, v. 4, n. 1, p. 95-114, 2010. DOI: <https://doi.org/10.1177/193758671000400107>.

WAGNER, Jennifer A. et al. Analyzing ICU patient room environmental quality through unoccupied, normal, and emergency procedure modes: an eqi evaluation. HERD: Health Environments Research \& Design Journal, v. 12, n. 4, p. 217-225, 2019. DOI:< http://dx.doi.org/10.1177/1937586719854218>. Acesso em: 4 abr. 2021.

WALDER, Bernhard et al. Effects of guidelines implementation in a surgical intensive care unit to control nighttime light and noise levels. Critical Care Medicine, v. 28, n. 7, p. 2242-2247, 2000. Disponível em: https://journals.Iww.com/ccmjournal/Abstract/2000/07000/Effects_of_guidelines_implementation _in_a_surgical.10.aspx. Acesso em: 19 jan. 2021.
Patrícia Biasi Cavalcanti patibiasi@yahoo.com

Camila Maçaneiro camila_macaneiro@hotmail.co m

Isabella Postiglione bellapostiglione@hotmail.com

Júlia Martan Nazário Palma juliamartanp@gmail.com

Júlia Roberta Eli julia.eli96@gmail.com 